\title{
Electronic cigarette use and indoor air quality in a natural setting
}

\author{
Eric K Soule, Sarah F Maloney, Tory R Spindle, Alyssa K Rudy, Marzena M Hiler, \\ Caroline 0 Cobb
}

Department of Psychology, Virginia Commonwealth University, Center for the Study of Tobacco Products, Richmond, Virginia, USA

\section{Correspondence to} Dr Eric K Soule, Virginia Commonwealth University, Department of Psychology, Center for the Study of Tobacco Products, P.O. Box 980205, Richmond VA 23298-0205, USA; eksoule@vcu.edu

Received 16 October 2015 Accepted 26 January 2016 Published Online First 15 February 2016

CrossMark

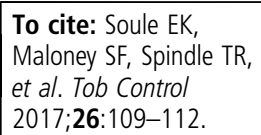

\section{ABSTRACT}

Introduction Secondhand smoke (SHS) from combustible cigarettes causes numerous diseases. Policies have been developed to prevent SHS exposure from indoor cigarette use to reduce health risks to non-smokers. However, fewer policies have been implemented to deter electronic cigarette (ECIG) use indoors, and limited research has examined the impact of secondhand exposure to ECIG aerosol.

Methods Indoor air quality was measured at a 2-day ECIG event held in a large room at a hotel. Fine particulate matter (PM) was measured using 2 devices that measured concentrations of PM $2.5 \mu \mathrm{m}$ aerodynamic diameter or smaller $\left(\mathrm{PM}_{2.5}\right)$. Measurements were taken before the event, over 2 days when the event was ongoing, and the day after the event. $\mathrm{PM}_{2.5}$ measurements were also taken from the restaurant at the hotel hosting the event and a restaurant at a nearby hotel.

Results During 6 time points when the event was ongoing, between 59 and 86 active ECIG users were present in the event room (room volume $=4023 \mathrm{~m}^{3}$ ). While the event was ongoing, median $\mathrm{PM}_{2.5}$ concentrations in the event room increased from a baseline of $1.92-3.20 \mu \mathrm{g} / \mathrm{m}^{3}$ to concentrations that ranged from $311.68 \mu \mathrm{g} / \mathrm{m}^{3}$ (IQR $253.44-411.84 \mu \mathrm{g} / \mathrm{m}^{3}$ ) to $818.88 \mu \mathrm{g} / \mathrm{m}^{3}$ (IQR $760.64-975.04 \mu \mathrm{g} / \mathrm{m}^{3}$ ).

Conclusions $\mathrm{PM}_{2.5}$ concentrations observed at the ECIG event were higher than concentrations reported previously in hookah cafés and bars that allow cigarette smoking. This study indicates that indoor ECIG use exposes non-users to secondhand ECIG aerosol. Regulatory bodies should consider establishing policies that prohibit ECIG use anywhere combustible cigarette use is prohibited.

\section{INTRODUCTION}

Exposure to secondhand smoke (SHS) from tobacco cigarettes (mainstream smoke exhaled by smokers and sidestream smoke emitted from the end of a burning cigarette) is known to cause a wide range of diseases including coronary heart disease and cancers. ${ }^{1}{ }^{2}$ To prevent non-smokers' risk of negative health consequences associated with SHS from tobacco cigarettes, over 4400 municipalities in the USA have enacted laws that restrict cigarette smoking, including approximately 800 municipalities that require all restaurants and bars to be $100 \%$ smoke free. ${ }^{3}$ More broadly, approximately 92 nations have enacted some type of $100 \%$ smoke-free law. ${ }^{4}$ As electronic cigarettes (ECIGs) continue to grow in popularity among adolescents $^{56}$ and adults, ${ }^{7}$ there is an increase in policies addressing exposure to secondhand ECIG aerosol. As of 2 October 2015, five US states and over 400 counties have implemented some form of restriction of ECIG use indoors. ${ }^{9}$ International policies are more varied with certain restrictions for ECIG use in UK airports and trains ${ }^{10}$ and reports of complete ECIG bans in indoor public places for Malta, Belgium and Spain. ${ }^{11}$

ECIGs are devices that use a heater to aerosolise a liquid often containing nicotine, propylene glycol, vegetable glycerin and flavourants. Research reveals that the aerosol produced from ECIG use contains toxicants including nicotine, glycols, aldehydes, metals, volatile organic compounds and polycyclic aromatic hydrocarbons. ${ }^{12}$ In addition, a growing body of work has examined the extent to which ECIG aerosol produced in 'exposure chambers' under controlled conditions by a single ECIG user $^{13-15}$ or in private homes ${ }^{16}$ influences indoor air quality. However, to our knowledge, no published studies have examined the extent to which ECIG aerosol production under natural use conditions in public venues influences indoor air quality. Addressing this issue may provide critical information to policymakers interested in protecting non-ECIG users from involuntary inhalation of ECIG aerosols. The purpose of this study was to examine the effect of ECIG use on indoor air quality in a natural setting.

\section{METHODS}

A research team attended an ECIG event (summer, 2015) held in a hotel meeting room over 2 days. ECIG use was permitted in all hotel areas. Indoor air quality (particulate matter measuring $<2.5 \mu \mathrm{m}$ $\left(\mathrm{PM}_{2.5}\right)$ ) was measured using two concealed TSI (Shoreview, Minnesota, USA) Sidepak AM510 Personal Aerosol Monitors in (1) the event room, (2) an indoor restaurant within the event hotel and (3) an indoor restaurant located at an adjacent hotel not hosting an ECIG event. The Sidepak AM510 measures PM by drawing ambient air into the device and analysing scattering light. This method has been used and validated previously for measuring secondhand tobacco smoke. ${ }^{17-21}$ (see also ref 15 and 22). $\mathrm{PM}_{2.5}$ was measured because it is a clinically relevant measure: particulates of this size are small enough to be deposited deep inside of the lung if inhaled and take longer to be removed by the body. ${ }^{23}$ The ease of concealment and ability to collect ambient air quality measurements in a natural environment make the Sidepak AM510 an appropriate choice for this type of assessment; however, this device cannot detect particle sizes below $100 \mathrm{~nm}$ in diameter. Because ECIG aerosol contains particles in this size range, ${ }^{14}$ 
the $\mathrm{PM}_{2.5}$ concentrations measured by the Sidepak AM510 likely underestimate the actual exposures being delivered to bystanders. However, using this device represented an appropriate option for the purpose of this study. Air quality measurements were collected in the event room the day before the event occurred, during the 2 days of the event at least two time points on each day, and the day after the event. Dimensions of each room where air quality was assessed were measured using a laser device, and these data were used to calculate room volume.

Prior to entering the venues, each Sidepak device was zerocalibrated according to device guidelines. Using methods similar to those reported elsewhere, ${ }^{22}$ a research team carrying a Sidepak device entered the venues and collected measurements for at least $30 \mathrm{~min}$. While in the venues, researchers sat at a table with the device at table height or walked around the rooms to collect air quality readings throughout the venue. Immediately prior to and after each measurement session, $5 \mathrm{~min}$ of control measurements were collected by sampling outdoor air or in a room with no active ECIG use. The total number of individuals and the number of active ECIG users in the venues were counted at multiple time points during each session and a session count average was calculated.

The Sidepak was set to average 60 consecutive $1 \mathrm{~s}$ measurements of $\mathrm{PM}_{2.5}$ (1 min log interval). The first and last readings of each session were omitted in analyses to prevent including air from outside of the venue while entering or exiting. $\mathrm{PM}_{2.5}$ readings measured during each session were ordered and averaged to obtain median and mean concentrations of $\mathrm{PM}_{2.5}$ in the venue ambient air. A calibration factor was applied to these readings to generate a more accurate value for the concentrations of the $\mathrm{PM}_{2.5}$. Previous research of ECIG aerosol has used a calibration factor of $0.32^{15}$ that has been identified for cigarette SHS. ${ }^{18} 1924$ Because the ECIG aerosol particle size profile includes particles with diameters below $100 \mathrm{~nm},{ }^{14}$ this calibration factor may underestimate $\mathrm{PM}_{2.5}$ concentration. However, in absence of an accepted calibration factor for ECIG aerosol, the cigarette SHS calibration factor was used. Additionally, active ECIG user density (defined as a visible ECIG in an individual's hand or mouth) was determined by calculating the number of ECIG users per $100 \mathrm{~m}^{3}$ in each venue. This determination was not possible during the event days because of the large number of individuals in the event room and size of the room (inability to reliably determine ECIG use in hand or mouth). Thus, all individuals in the main event room during the event days were considered active ECIG users for the purposes of calculating active ECIG density.

\section{RESULTS}

As displayed in table 1 , the event room was a large space $\left(4023 \mathrm{~m}^{3}\right)$ compared with the hotel restaurants that were included in the study $\left(596\right.$ and $942 \mathrm{~m}^{3}$ ). Cigarette smoking was not allowed inside any of the included venues; however, ECIG use was observed in the main ECIG event room (before, during and after the event) and the restaurant in the hotel hosting the event. During the event, 59-86 active ECIG users were observed at six different time points.

Table 1 shows that the day before the event the median and mean $\mathrm{PM}_{2.5}$ concentrations in the main ECIG event room were less than $5 \mu \mathrm{g} / \mathrm{m}^{3}$ and both devices measured within $2 \mu \mathrm{g} / \mathrm{m}^{3}$ between device 1 (median $=1.92 \mu \mathrm{g} / \mathrm{m}^{3}$, mean $\left.=2.08 \mu \mathrm{g} / \mathrm{m}^{3}\right)$ and device 2 (median $=3.20 \mu \mathrm{g} / \mathrm{m}^{3}$, mean $\left.=3.19 \mu \mathrm{g} / \mathrm{m}^{3}\right)$. These control readings were of the same magnitude as the samples from the hotel restaurants (median $=1.60-5.76 \mu \mathrm{g} / \mathrm{m}^{3}$, mean $\left.=1.72-5.89 \mu \mathrm{g} / \mathrm{m}^{3}\right)$. When the event was ongoing, median $\mathrm{PM}_{2.5}$ concentrations in the event room ranged from $311.68 \mu \mathrm{g} /$ $\mathrm{m}^{3} \quad$ (IQR $253.44-411.84 \mu \mathrm{g} / \mathrm{m}^{3}, \quad$ mean $=330.97 \mu \mathrm{g} / \mathrm{m}^{3}$, $\mathrm{SD}=88.18$ ) to $818.88 \mu \mathrm{g} / \mathrm{m}^{3} \quad$ (IQR $760.64-975.04 \mu \mathrm{g} / \mathrm{m}^{3}$, mean $=869.12 \mu \mathrm{g} / \mathrm{m}^{3}, \mathrm{SD}=139.29$; see table 1) with average median concentrations of $595.31 \mu \mathrm{g} / \mathrm{m}^{3}$ (average mean concentration $=607.12 \mu \mathrm{g} / \mathrm{m}^{3}$ ) over the six time points measured. Approximately $17 \mathrm{~h}$ after the ECIG event concluded, the

Table 1 Descriptive statistics and $\mathrm{PM}_{2.5}$ concentration for venues measured at different time points

\begin{tabular}{|c|c|c|c|c|c|c|c|c|c|}
\hline Venue & Day of week & Time of day & $\begin{array}{l}\text { Device } \\
\text { number }\end{array}$ & Volume $\left(\mathrm{m}^{3}\right)$ & $\begin{array}{l}\text { ECIG use } \\
\text { observed }\end{array}$ & $\begin{array}{l}\text { Active ECIG } \\
\text { users }\end{array}$ & $\begin{array}{l}\text { Active ECIG user } \\
\text { density* }\end{array}$ & $\begin{array}{l}\text { Mean } \mathrm{PM}_{2.5} \\
\left(\mu \mathrm{g} / \mathrm{m}^{3}\right)\end{array}$ & $\begin{array}{l}\text { Median } \mathrm{PM}_{2.5} \\
\left(\mu \mathrm{g} / \mathrm{m}^{3}\right)\end{array}$ \\
\hline \multicolumn{10}{|c|}{ ECIG event room } \\
\hline & Thursday (pre-event) & $12: 58-13: 28$ & 1 & 4023 & Yes & 0 & 0 & 2.09 & 1.92 \\
\hline & Thursday (pre-event) & $12: 58-13: 28$ & 2 & 4023 & Yes & 0 & 0 & 3.19 & 3.20 \\
\hline & Friday (event day 1) & 14:54-15:15 & 1 & 4023 & Yes & $61 \dagger$ & 1.52 & 786.81 & 769.60 \\
\hline & Friday (event day 1) & $18: 31-19: 02$ & 1 & 4023 & Yes & $86+$ & 2.14 & 689.88 & 703.04 \\
\hline & Saturday (event day 2) & $14: 45-15: 15$ & 2 & 4023 & Yes & $59+$ & 1.47 & 869.12 & 818.88 \\
\hline & Saturday (event day 2) & $15: 17-15: 48$ & 1 & 4023 & Yes & $66 t$ & 1.64 & 556.17 & 584.64 \\
\hline & Saturday (event day 2) & 18:00-18:30 & 1 & 4023 & Yes & $76+$ & 1.89 & 409.78 & 384.00 \\
\hline & Saturday (event day 2) & $18: 30-19: 00$ & 2 & 4023 & Yes & $76 t$ & 1.9 & 330.97 & 311.68 \\
\hline & Sunday (post-event) & $12: 45-13: 15$ & 1 & $2827 \ddagger$ & Yes & 0 & 0 & 12.85 & 12.80 \\
\hline & Sunday (post-event) & $12: 45-13: 15$ & 2 & $2827 \ddagger$ & Yes & 0 & 0 & 15.82 & 15.52 \\
\hline \multicolumn{10}{|c|}{ Hotel restaurant 1} \\
\hline & Thursday (pre-event) & $13: 42-14: 27$ & 1 & 596 & Yes & 2 & 0.34 & 1.72 & 1.60 \\
\hline & Thursday (pre-event) & $13: 42-14: 27$ & 2 & 596 & Yes & 2 & 0.34 & 2.49 & 2.56 \\
\hline & Friday (event day 1) & 19:14-20:07 & 1 & 596 & No & 0 & 0 & 4.92 & 4.80 \\
\hline \multicolumn{10}{|c|}{ Hotel restaurant 2} \\
\hline & Friday (event day 1) & $20: 21-20: 51$ & 1 & 942 & No & 0 & 0 & 5.89 & 5.76 \\
\hline
\end{tabular}


median $\mathrm{PM}_{2.5}$ concentration in the event room was 12.80 $15.52 \mu \mathrm{g} / \mathrm{m}^{3}$ (mean $12.85-15.82 \mu \mathrm{g} / \mathrm{m}^{3}$ ) across both devices.

Figure 1 displays 1 min average $\mathrm{PM}_{2.5}$ concentrations in the event room pre-event (device 2), during day 1 of the event (device 1) and post-event (device 1).

\section{DISCUSSION}

In this study, the presence of fine PM increased dramatically125-330 times higher-in a room where active ECIG use was occurring relative to the same room when no active ECIG use was occurring or in other venues where no active ECIG use was occurring. This observation indicates that indoor ECIG use can generate fine PM in high concentrations during natural use conditions in indoor environments. The $\mathrm{PM}_{2.5}$ concentrations observed in this study were approximately four times higher than $\mathrm{PM}_{2.5}$ generated by single ECIG users who used their device twice for $5 \mathrm{~min}$ in an exposure chamber $\left(151.7 \mu \mathrm{g} / \mathrm{m}^{312}\right)$ and more than 60 times higher than median $\mathrm{PM}_{2.5}$ measured inside the home of an ECIG user. ${ }^{16}$ Particulate concentrations observed in this study were greater than $\mathrm{PM}_{2.5}$ concentrations found in hookah cafés and indoor bars that allow cigarette smoking (eg, mean $\mathrm{PM}_{2.5}$ concentrations from 17 hookah cafés of $369-384 \mu \mathrm{g} / \mathrm{m}^{3}$ and from bars that allowed cigarette smoking of $119 \mu \mathrm{g} / \mathrm{m}^{322}$ ). Additionally, the $\mathrm{PM}_{2.5}$ concentrations from this study are likely higher still compared with those reported previously $y^{22}$ because the $\mathrm{PM}_{2.5}$ concentrations reported in this study may underestimate the actual $\mathrm{PM}_{2.5}$ concentrations due to device measurement limitations and applying a calibration factor used to examine SHS from combustible cigarettes.

While ECIG aerosol often contains some of the same chemicals found in combustible cigarette or hookah smoke such as nicotine, the composition (ie, concentration of each chemical per puff or product use) of the PM measured in this study likely differs from PM generated from combustible cigarette and hookah smoking. ${ }^{14} 1525$ Therefore, this study does not provide the data needed for a direct comparison of the harms associated with exposure to high concentrations of PM generated from ECIG use and hookah or combustible cigarette smoke. While the exact harm potential of secondhand exposure to ECIG aerosol is not currently known, the fact that secondhand ECIG aerosol contains fine particulates, nicotine, carcinogenic aldehydes, polycyclic aromatic hydrocarbons and volatile organic

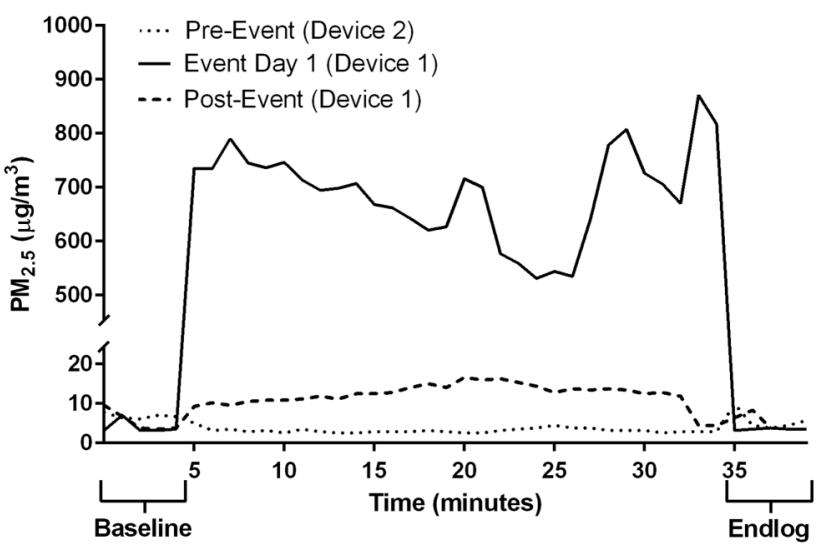

Figure 1 Representative particulate matter measuring $<2.5 \mu \mathrm{m}$ $\left(\mathrm{PM}_{2.5}\right)$ concentrations in $\mathrm{ECIG}$ event room measured before the event (pre-event), during the event (event day 1) and after the event (postevent). Each of these representative measurement sessions includes 5 baseline readings outside of event room, 30 readings in the event room and 5 endlog readings outside of event room. compounds ${ }^{13-16}$ indicates that exposure to secondhand ECIG aerosol may present some degree of harm to bystanders. Importantly, any of these bystanders who are not ECIG users have opted not to inhale ECIG aerosols yet, should they share indoor space with an ECIG user who is using their ECIG actively, are involuntarily inhaling these fine particulates.

This study is the first to examine ECIG-generated PM in a natural public setting. While the venue examined in this study may differ from some places where ECIGs are used indoors commonly, we expect that $\mathrm{PM}_{2.5}$ concentrations measured in other venues, such as bars or restaurants that allow ECIG use, would be elevated similarly compared with the concentrations reported in this study, again requiring non-users to inhale fine PM involuntarily. This involuntary inhalation unfortunately could be the norm if policies are not established that prevent ECIG use in airplanes, hotels, hospitals, schools, restaurants, bars and other venues were cigarette smoking was once commonplace in this country. Policymakers may want to consider to what extent ECIG users, like cigarette smokers, are required to comply with all existing clean indoor air regulations.

\section{What this paper adds}

This is the first study to examine the effect of electronic cigarette (ECIG) use on indoor air quality in a natural setting. Particulate matter (PM) measuring $<2.5 \mu \mathrm{m}$ concentrations in a large room with active ECIG users were higher than those previously reported in hookah cafés and bars that allow cigarette smoking. While the harm potential of secondhand exposure to ECIG exposure among non-ECIG users is unknown currently, these data indicate that indoor ECIG can affect indoor air quality. Policies that prohibit indoor ECIG use would prevent non-ECIG users' non-voluntary inhalation of ECIG-generated PM.

Contributors EKS and COC made significant contributions to the study design data analysis and writing of the manuscript. SFM, TRS, AKR, MMH and COC conducted all data collection and provided critical review of the manuscript. All authors approved the final version of the manuscript.

Funding Research reported in this publication was supported by the National Institute on Drug Abuse of the National Institutes of Health under Award Number P50DA036105 and the Center for Tobacco Products of the US Food and Drug Administration.

Disclaimer The content is solely the responsibility of the authors and does not necessarily represent the official views of the National Institutes of Health or the Food and Drug Administration.

Competing interests None declared.

Provenance and peer review Not commissioned; externally peer reviewed.

Data sharing statement This manuscript contains all data collected for this study. Data are available by email request from the corresponding author (EKS).

\section{REFERENCES}

1 US Department of Health and Human Services. The health consequences of involuntary exposure to tobacco smoke: a report of the Surgeon General. Atlanta, GA: U.S. Department of Health and Human Services, Centers for Disease Control and Prevention, National Center for Chronic Disease Prevention and Health Promotion, Office on Smoking and Health, 2006.

2 National Cancer Institute. Health effects of exposure to environmental tobacco smoke. Smoking and tobacco control monograph No. 10. 1999, NIH Pub. No. 99-4645. http://cancercontrol.cancer.gov/tcrb/monographs/10/index.html (accessed 5 Oct 2015).

3 American Nonsmokers' Rights Foundation. Overview list-How many smoke free laws? http://www.no-smoke.org/pdf/mediaordlist.pdf (accessed 5 Oct 2015). 
4 American Nonsmokers' Rights Foundation. Smokefree status of workplaces and hospitality venue around the world. http://www.no-smoke.org/pdf/ internationalbarsandrestaurants.pdf (accessed 27 Dec 2015).

5 Centers for Disease Control and Prevention. Tobacco product use among middle and high school students-United States, 2011 and 2012. MMWR Morb Mortal Wkly Rep 2013;62:893-7.

6 Arrazola RA, Neff LJ, Kennedy SM, et al. 2014. Tobacco use among middle and high school students-United States, 2013. MMWR Morb Mortal Wkly Rep 2014;63:1021-6.

7 McMillen RC, Gottlieb MA, Shaefer RM, et al. Trends in electronic cigarette use among U.S. adults: use is increasing in both smokers and nonsmokers. Nicotine Tob Res 2015;17:1195-202.

8 King BA, Patel R, Nguyen $\mathrm{KH}$, et al. Trends in awareness and use of electronic cigarettes among US adults, 2010-2013. Nicotine Tob Res 2015;17:219-27.

9 American Nonsmokers' Rights Foundation. States and municipalities with laws regulating use of electronic cigarettes. http://www.no-smoke.org/pdf/ecigslaws.pdf (accessed 12 Oct 2015).

10 BBC. So where can you still vape? BBC Newsbeat 10 June 2015. http://www.bbc. co.uk/newsbeat/article/33079473/so-where-can-you-still-vape (accessed 27 Dec 2015).

11 Deans D. E-cigarettes 'set to be banned' indoors in public places from next year. Mirror 9 June 2015. http://www.mirror.co.uk/news/uk-news/ e-cigarettes-set-banned-indoors-public-5850168 (accessed 27 Dec 2015).

12 Cheng T. Chemical evaluation of electronic cigarettes. Tob Control 2014;23(Suppl 2):ii11-17.

13 Schober W, Szendrei K, Matzen W, et al. Use of electronic cigarettes (e-cigarettes) impairs indoor air quality and increases FeNO levels of e-cigarette consumers. Int J Hyg Environ Health 2014;217:628-37.

14 Schripp T, Markewitz D, Uhde E, et al. Does e-cigarette consumption cause passive vaping? Indoor Air 2013;23:25-31.
15 Czogala J, Goniewicz ML, Fidelus B, et al. Secondhand exposure to vapors from electronic cigarettes. Nicotine Tob Res 2014;16:655-62.

16 Fernández $E$, Ballbè $M$, Sureda $X$, et al. Particulate matter from electronic cigarettes and conventional cigarettes: a systematic review and observational study. Curr Environ Health Rep 2015;2:423-29.

17 Centers for Disease Control and Prevention. Indoor air quality in hospitality venues before and after the implementation of a clean indoor air law-Western New York, 2003. MMWR Morb Mortal Wkly Rep 2004;53:1038-41.

18 Lee K, Hahn EJ, Pieper N, et al. Differential impacts of smoke-free laws on indoor air quality. J Environ Health 2008;70:24-30, 54.

19 Klepeis NE, Ott WR, Switzer P. Real-time measurement of outdoor tobacco smoke particles. J Air Waste Manag Assoc 2007;57:522-34.

20 Hyland A, Travers MJ, Dresler C, et al. A 32-country comparison of tobacco smoke derived particle levels in indoor public places. Tob Control 2008;17:159-65

21 Waring MS, Siegel JA. An evaluation of the indoor air quality in bars before and after a smoking ban in Austin, Texas. J Expo Sci Environ Epidemiol 2007; $17: 260-8$

22 Cobb CO, Vansickel AR, Blank MD, et al. Indoor air quality in Virginia waterpipe cafés. Tob Control 2013;22:338-43.

23 Heyder J. Deposition of inhaled particles in the human respiratory tract and consequences for regional targeting in respiratory drug delivery. Proc Am Thorac Soc 2004; 1:315-20.

24 Travers MJ. Smoke-free air policy: changing what's in the air and in the body, in social and preventive medicine. [Dissertation]. Buffalo, NY: State University of New York at Buffalo, 2008.

25 Daher N, Saleh R, Jaroudi E, et al. Comparison of carcinogen, carbon monoxide, and ultrafine particle emissions from narghile waterpipe and cigarette smoking: sidestream smoke measurements and assessment of second-hand smoke emission factors. Atmos Environ 2010;44:8-14. 